\title{
H19 long noncoding RNA alters trophoblast cell migration and invasion by regulating T $\beta R 3$ in placentae with fetal growth restriction
}

\author{
Lisa Zuckerwise ${ }^{1, *}$, Jing $\mathrm{Li}^{1,2, *}$, Lingeng $\mathrm{Lu}^{3}$, Yi Men ${ }^{1,4}$, Tingting Geng ${ }^{1,5}$, Catalin \\ S. Buhimschi ${ }^{6}$, Irina A. Buhimschi ${ }^{6,7,8}$, Radek Bukowski ${ }^{1}$, Seth Guller ${ }^{1}$, Michael \\ Paidas ${ }^{1,9}$, Yingqun Huang ${ }^{1}$ \\ ${ }^{1}$ Department of Obstetrics, Gynecology and Reproductive Sciences, Yale University School of Medicine, New Haven, CT, USA \\ ${ }^{2}$ Deparment of Obstetrics and Gynecology, Nanfang Hospital, Southern Medical University, Guangzhou, Guangdong, P.R. China \\ ${ }^{3}$ Department of Chronic Diseases Epidemiology, Yale School of Public Health, Yale University School of Medicine, New Haven, \\ CT, USA \\ ${ }^{4}$ Department of Head and Neck Surgery, State Key Laboratory of Oral Diseases, West China Hospital of Stomatology, Sichuan \\ University, Chengdu, Sichuan, P.R. China \\ ${ }^{5}$ Department of Endocrinology, School of Medicine, First Affiliated Hospital of Xi'an Jiaotong University, Xi'an, Shaanxi, P.R. \\ China \\ ${ }^{6}$ Department of Obstetrics and Gynecology, The Ohio State University College of Medicine, Columbus, OH, USA \\ ${ }^{7}$ Center for Perinatal Research, The Research Institute at Nationwide Children's Hospital, Columbus, OH, USA \\ ${ }^{8}$ Department of Pediatrics, The Ohio State University College of Medicine, Columbus, OH, USA \\ 'Yale Women and Children's Center for Blood Disorders and Preeclampsia Advancement, Yale School of Medicine, New \\ Haven, CT, USA \\ *These authors contributed equally to this work
}

Correspondence to: Yingqun Huang, email: yingqun.huang@yale.edu

Keywords: fetal growth restriction, $\mathrm{H} 19$ long noncoding RNA, TRR3, trophoblast

Received: February 15, $2016 \quad$ Accepted: May 08, $2016 \quad$ Published: May 21, 2016

\section{ABSTRACT}

Fetal growth restriction (FGR) is a well-recognized risk factor for perinatal mortality and morbidity, as well as neurodevelopmental impairment and adulthood onset disorders. Here we report that the H19 long noncoding RNA (IncRNA) is significantly decreased in placentae from pregnancies with FGR. Downregulation of H19 leads to reduced migration and invasion of extravillous trophoblast (EVT) cells in vitro. This is consistent with reduced trophoblast invasion that has been observed in FGR. Genome-scale transcriptome profiling of EVT cells reveals significantly decreased expression of the type III TGF- $\beta$ receptor (T $\beta R 3$ ) following $\mathrm{H} 19$ knockdown. Decreased $T \beta R 3$ expression is also seen in FGR placentae. T $\beta R 3$ repression decreases EVT cell migration and invasion, owing to impaired TGF- $\beta$ signaling through a non-canonical TGF- $\beta$ signaling pathway. Further, we identify T $\beta R 3$ as a novel regulatory target of microRNA let-7. We propose that dysregulation of this newly identified H19/TßR3mediated regulatory pathway may contribute to the molecular mechanism of FGR. Our findings are the first to show a IncRNA-based mechanism of FGR, holding promise for the development of novel predictive, diagnostic, and therapeutic modalities for FGR.

\section{INTRODUCTION}

Fetal growth restriction (FGR), defined as estimated fetal weight below the 10th percentile, identifies a group of fetuses who are at risk for perinatal and longer term mortality and morbidity $[1,2]$. Perinatal morbidity associated with FGR includes neonatal encephalopathy, cerebral palsy, sepsis, seizures, respiratory distress, prolonged hospitalization and need for intensive care unit admission. These risks increase with severity of growth restriction. In preterm infants with FGR, a large retrospective study of over one million neonates found an increased risk of 
respiratory distress syndrome, intraventricular hemorrhage, and necrotizing enterocolitis as compared to appropriately sized gestational-age matched neonates [3]. The increased morbidity associated with FGR extends beyond the neonatal period. FGR is associated with increased risk of cardiovascular and endocrine disorders in adulthood [4]. Additionally, children from pregnancies with FGR have demonstrated significantly lower academic achievement in school as well as lower professional achievement in adulthood [5,6]. The molecular mechanisms underlying FGR remain in the majority of cases poorly understood.

Proper placental development is essential for fetal growth and survival. The extravillous trophoblast (EVT), a subset of placental cells, plays a critical role in this process. EVT cells migrate and invade into the uterine wall, leading to remodeling of the maternal vasculature. This remodeling yields a low-resistance, high-capacity perfusion system that allows for adequate exchange of oxygen, nutrients and key molecules at the maternal-fetal interface. The migratory and invasive properties of EVT are stringently controlled and derangement can lead to pathological conditions. Indeed, suboptimal trophoblast invasion has been associated with FGR and preeclampsia, whereas excessive trophoblast invasion has been linked to placenta accreta and choriocarcinoma (reviewed in $[7,8]$ ).

The $H 19$ gene encodes a polyadenylated, long noncoding RNA (lncRNA) of 2,600-nt that predominantly resides in the cytoplasm but with a minor fraction also found in the nucleus $[9,10]$. H19 is abundantly expressed both in placentae and embryos, and is strongly down-regulated after birth in most adult tissues [9]. Mechanistically, it has been shown that H19 lncRNA (herein called H19) in the nucleus recruits repressive histone markers to differentially methylated regions of a group of imprinted network genes, thereby inhibiting their transcription and contributing to embryo growth regulation in the mouse [10]. Nuclear H19 also serves as precursor for microRNA miR-675 in a cell/ tissue-specific and developmentally regulated manner [11-13]. In the mouse, through the action of miR-675, H19 maintains adult hematopoietic stem cells [12], promotes skeletal muscle differentiation and regeneration [13], and results in the physiological inhibition of placental growth just before birth [11]. In the cytoplasm, H19 acts as a molecular sponge for microRNA let-7 thereby reducing its bioavailability and preventing it from repressing target gene expression at the posttranscriptional level [14]. Through this action, H19 plays a role in regulation of muscle cell differentiation [14], glucose metabolism [15], tumor metastasis [16], and endometrial development [17].

Altered imprinting or epigenetic regulation of the H19-Igf2 locus in human has been associated with altered placental and fetal growth as well as pregnancy complications ([18, 19], and reviewed in [20]). However, the underlying molecular mechanism of H19's role in placentation remains poorly defined. Using in situ hybridization it was shown that $H 19$ is highly expressed in human placental intermediate trophoblast, cytotrophoblast (including EVT), and syncytiotrophoblast tissue [21], suggesting an important role for H19 in trophoblast physiology.

In this report, we investigate a mechanism through which H19 participates in the pathogenesis of FGR. We find that H19 is significantly decreased in human placentae with idiopathic FGR. We provide evidence that depressed H19 reduces TGF- $\beta$ signaling through a non-canonical pathway activated by $T \beta R 3$, leading to impaired migration and invasion of EVT cells. We propose that dysregulation of this newly identified H19/T $\beta$ R3-mediated regulatory pathway may contribute to the underlying mechanism of idiopathic FGR.

\section{RESULTS}

\section{H19 promotes EVT migration and invasion by inhibiting microRNA let-7}

We have previously reported that H19 promotes migration and invasion of tumor cells by decreasing the bioavailability of microRNA let-7 [16]. Bearing multiple binding sites for let-7, H19 binds to and sequesters let-7, preventing it from repressing target gene expression at the posttranscriptional level [14]. Given the abundance of H19 in the EVT [21], and its known role in regulating migration and invasion, we hypothesized that H19 might function to regulate EVT development. Thus, effects of $\mathrm{H} 19$ repression on EVT were evaluated using HTR-8/SVneo (called HTR herein), a cell line derived from human first trimester EVT [22]. H19 was knocked down using siRNA (siH19, [14, 16]) in the presence and absence of a let-7 inhibitor (iLet7, [14, $16]$ ), followed by analysis of cell migration and invasion using transwell assays. iLet7s are chemically modified oligonucleotides that specifically bind to let-7 and inhibit its activity. The rationale for including iLet7 was to confirm the contribution of let-7 to the H19-mediated pathway, as H19 has other functions besides sequestering let-7 [10-13, 23]. Thus, in the presence of iLet7, we would expect that the effects of H19 knockdown on EVT would be attenuated, as iLet7 would neutralize let-7 released from H19 sequestration. The ability of iLet7 to relieve inhibition of other let-7 targets has been previously documented [15-17].

When H19 was downregulated by siH19 (Figure 1A, compare middle column to left column), there was a concomitant decrease in cell migration (Figure 1B, compare middle column to left column) and invasion (Figure $1 \mathrm{C}$, compare middle column to left column). The decreases in cell migration and invasion were not due to decreased cell proliferation and/or increased cell death (Supplementary Figure 1A). A combination of iLet7 and H19 knockdown (Figure 1A, compare right column to left column) restored both migration (Figure 1B, right column) and invasion (Figure 1C, right column) to control levels. Next, we performed reciprocal experiments by overexpressing H19. 
We transfected HTR with an H19-expressing plasmid pH19 $[14,16]$ or an empty vector as a negative control. H19 overexpression (Figure 1D) led to an increase both in migration (Figure 1E) and invasion (Figure 1F) which was not due to either increased cell proliferation and/or decreased cell death (Supplementary Figure 1B). Collectively, these results suggested that $\mathrm{H} 19$ promotes migration and invasion of EVT cells and that this regulation is achieved at least in part by reducing the bioavailability of let- 7 .

\section{TßR3 is a target of the H19/let-7 axis}

To identify downstream genes that mediate the H19/let-7-dependent regulation of EVT, we took a genome-wide approach. HTR cells were transfected with siCon and siH19, and RNAs were extracted $48 \mathrm{~h}$ later and subjected to high throughput RNA deep sequencing (RNA-seq). Among the numerous genes that showed significantly altered expression as a result of H19 repression (Supplementary Data 1, and Gene Expression Omnibus (GEO) accession number GSE80237) was the type III TGF- $\beta$ receptor (T $\beta$ R3, also called betaglycan).
The family of TGF- $\beta$ cytokines (including TGF- $\beta 1$, TGF- $\beta 2$, and TGF- $\beta 3$ ) signal by activating a receptor heterotetramer composed of two T $\beta R 1: T \beta R 2$ heterodimers [24] which subsequently phosphorylate (through T $\beta R 1$ 's cytoplasmic kinase domain) the SMAD proteins which then move into the nucleus to orchestrate transcriptional responses [25]. T $\beta R 3$ is unique in that its cytoplasmic domain lacks a kinase activity and that it functions as a direct regulator of TGF- $\beta$ access to the signaling receptors. T $\beta$ R3 binds and presents TGF- $\beta$ to the T $\beta R 1: T \beta R 2$ complexes, thereby augmenting cell responsiveness to TGF- $\beta$ [26]. We decided to focus on T $\beta$ R3 for several reasons. First, TGF- $\beta$ signaling is known to regulate EVT migration and invasion (reviewed in $[8,27]$ ), but a role for T $\beta R 3$ in these processes has not been documented. Second, human placental trophoblast cells in primary culture have been found to possess a predominant level of T $\beta R 3$ and relatively lower levels of T $\beta R 1$ and T $\beta R 2$ [28]. Third, T $\beta$ R3 has been shown to regulate invasion of non-placental cells. For example, T $\beta$ R3 enhances invasion of epicardial cells through activating the Par6/Smurf1/ RhoA pathway independent of SMADs [29], whereas loss
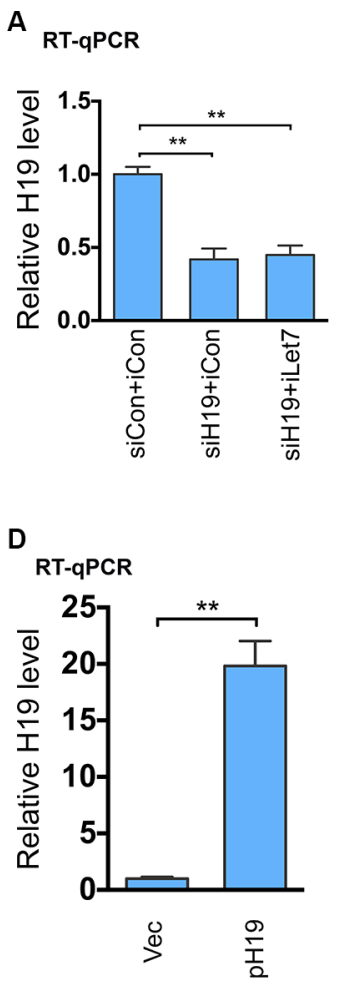

B Migration

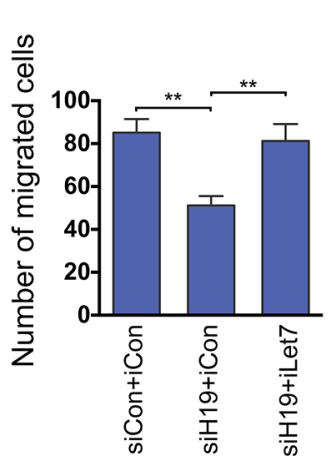

E

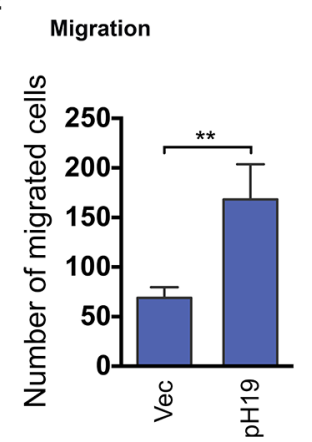

C
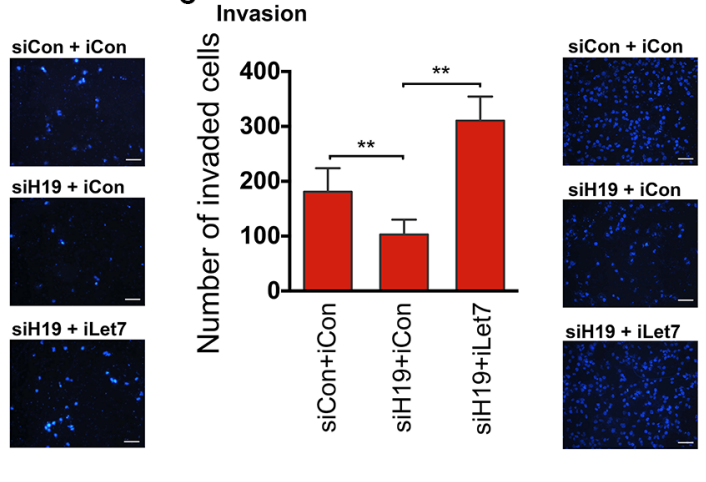

$\mathbf{F}$
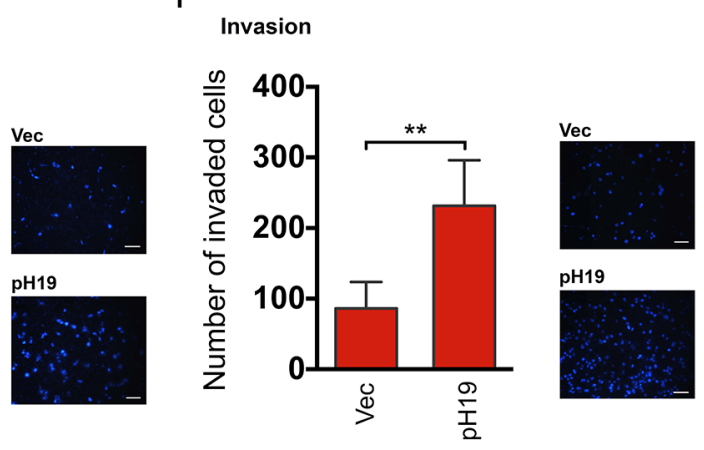

Figure 1: The H19/let-7 axis regulates trophoblast cell migration and invasion. (A-C) HTR cells were transfected with siCon (control siRNA) plus iCon (microRNA inhibitor control), siH19 (H19-specific siRNA) plus iCon, or siH19 plus iLet-7 (let-7-specific inhibitor). RNAs were extracted $48 \mathrm{~h}$ post-transfection and analyzed by RT-qPCR. Relative H19 levels after normalization against betatubulin mRNA are presented in A. For cell migration and invasion analyses, $48 \mathrm{~h}$ following transfection, equal numbers of cells from each group were seeded into upper chambers. Migration and invasion were allowed to occur for $20 \mathrm{~h}$ and $36 \mathrm{~h}$, respectively. Group $\mathrm{t}$ tests were performed to compare each data point with the control (siCon+iCon). Numbers are mean $\pm \mathrm{SD}(n=3)$. ${ }^{*} p<0.01$. Representative Dapistained images of migrated (B) and invaded (C) cells are presented on the right. Original magnification: $\times 100$. Size bar: $50 \mu \mathrm{m}$. (D-F) HTR cells were transfected with empty vector or $\mathrm{pH} 19$ (H19-expressing plasmid). RNAs were extracted $48 \mathrm{~h}$ post-transfection and analyzed by RT-qPCR (D). Results of cell migration (E) and invasion (F) are presented. Group t tests were performed to compare each data point with the control (Vec). Numbers are mean $\pm \mathrm{SD}(n=3) . * *<0.01$. 
of T $\beta \mathrm{R} 3$ results in decreased mesenchymal cell invasion [30]. Finally, downregulation of T $\beta$ R3 expression was seen in H19 knockdown EVT cells and also in human FGR placentae where both H19 and T $\beta$ R3 significantly decreased as compared to control placentae (see below).

When H19 was downregulated (Figure 2A, left column, compare red bar with green bar), the level of T $\beta$ R3 mRNA also decreased (middle column), while that of T $\beta R 1$ mRNA was not affected (right column). Importantly, iLet7 was able to partially rescue T $\beta R 3$ mRNA level to that of the control (middle column, compare blue bar to red bar), consistent with T $\beta$ R3 being a target of let-7 inhibition. The incomplete restoration of T $\beta$ R3 mRNA level by iLet7 suggested additional regulatory mechanisms underlying T $\beta \mathrm{R} 3$ expression (see Discussion). Indeed, bioinformatic analysis predicted binding sites for multiple let-7 family microRNAs including let-7g in the $3^{\prime}$-UTR of T $\beta R 3$ (Figure $2 \mathrm{~B}$ and Supplementary Figure 2). Transfection of let-7 into HTR cells destabilized T $\beta$ R3 mRNA (Figure 2C, top panel) but not the negative control beta-actin mRNA (bottom panel), consistent with microRNA-induced mRNA target degradation [31]. Let-7-mediated inhibition of T $\beta R 3$ expression was further confirmed by Western blot analysis of cells transfected with let-7 (Supplementary Figure 3). Taken together, these results suggested that $T \beta R 3$ is a target of post-transcriptional regulation by the H19/let-7 axis in EVT cells.

\section{T $\beta R 3$ affects EVT migration and invasion through a non-canonical TGF- $\beta$ signaling}

To confirm that T $\beta \mathrm{R} 3$ is a key downstream mediator of the H19/let-7 axis, T $\beta$ R3 knockdown experiments were carried out in HTR cells and effects were evaluated. When T $\beta R 3$ was reduced at both the mRNA (Figure 3A) and protein (Figure 3B) levels using a previously validated siRNA [32], cell migration (Figure 3C) and invasion (Figure 3D) also decreased without affecting cell viabilities (Supplementary Figure 4). These findings suggest that T $\beta$ R3 positively impacts EVT migration and invasion. To determine the signal transduction pathway underlying the T $\beta R$ 3-dependent regulation, we first looked into the canonical SMAD pathway [25]. We observed no changes in SMAD phosphorylation upon T $\beta$ R3 repression in HRT cells (Supplementary Figure 5). We next investigated the possible involvement of the non-canonical Par6/Smurf1/ RhoA pathway previously shown to mediate TGF- $\beta 2$ induced, T $\beta R 3$-dependent epicardial cell invasion [29]. Upon TGF- $\beta$ stimulation, T $\beta$ R2 phosphorylates Par6, a key regulator of cell polarity and tight junction assembly. Activated Par6 recruits the E3 ubiquitin ligase Smurf1,
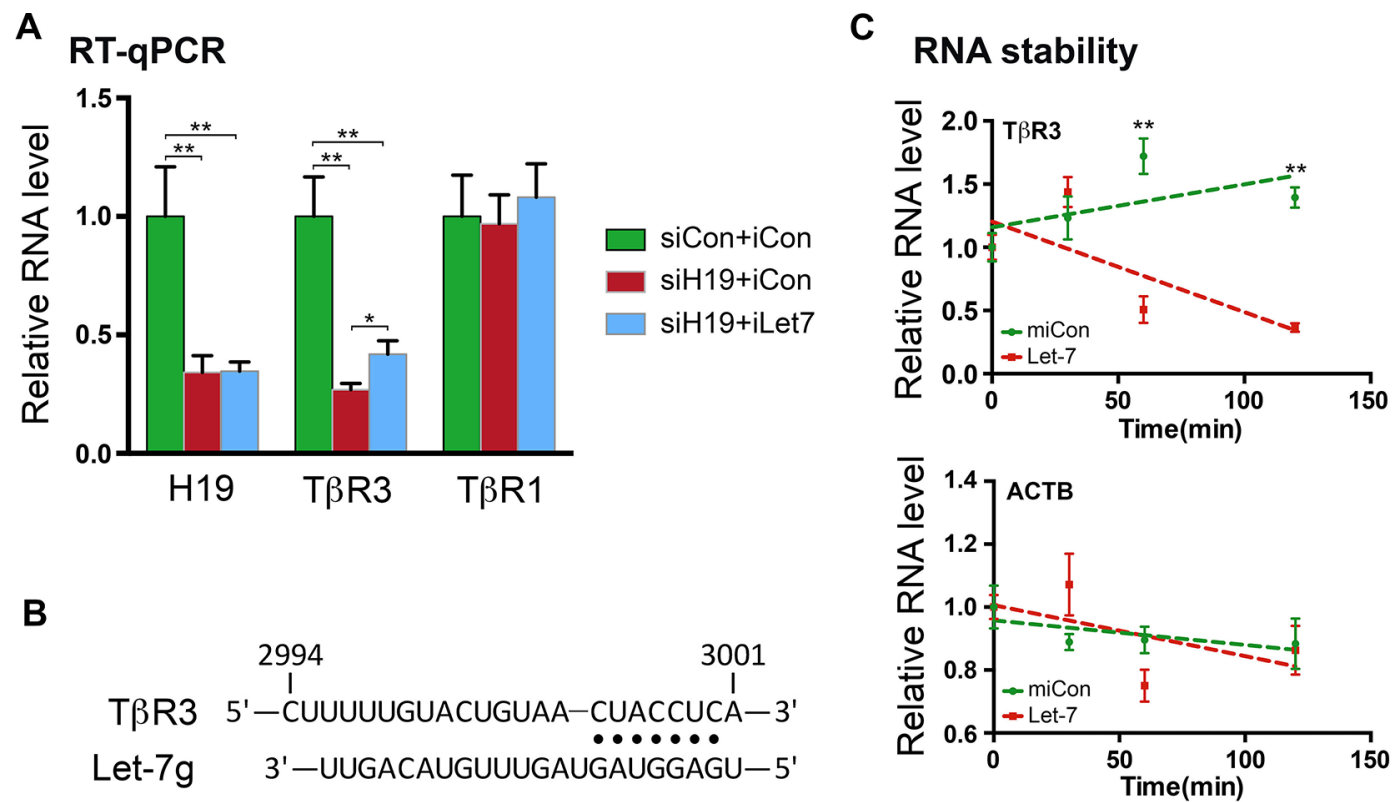

Figure 2: TßR3 is a target of the H19/let-7-mediated regulation. (A) HTR cells were transfected with siCon plus iCon, siH19 plus iCon or siH19 plus iLet7. RNAs were extracted $48 \mathrm{~h}$ later and analyzed by RT-qPCR. The indicated RNA levels relative to those of the control (siCon+iCon, which were arbitrarily set as 1) are presented. One-sample $t$ tests were performed to compare each data point with the siCon $+\mathrm{iCon}$ control. Numbers are mean $\pm \mathrm{SD}(n=3) . * *<0.01$. (B) Schematic presentation of let-7g-binding site in T $\beta R 3$ mRNA 3'-UTR. Partial sequence of human T $\beta R 3$ (top) and the sequence of let-7g (bottom) are shown. Base-paired interactions between the microRNA seed region (position 2 to 8 ) and the target mRNA are indicated by dots. Numbers are in nucleotides relative to the transcriptional start site of T $\beta$ R3. (C) HTR cells were transfected with Let-7 or miCon in the presence of the RNA polymerase inhibitor actinomycin D. RNAs were extracted at $0,30,60$, and 120 minutes following incubation with the transfection mixture. T $\beta R 3$ (upper panel) and beta-actin mRNA (bottom panel) levels were determined by RT-qPCR. Results are presented after normalization against beta-tubulin mRNA. Numbers are mean $\pm \mathrm{SD}(n=3)$. ${ }^{* *} p<0.01$. 
which then targets RhoA for degradation [33, 34]. The Rho GTPase RhoA regulates cell motility through activation of a variety of downstream effector proteins [35]. Importantly, activation of the Par6/Smurf1/RhoA pathway which leads to RhoA degradation has been shown to be required for maintaining tumor cell motility and epicardial cell invasion [29, 33]. As T $\beta$ R3 knockdown reduces EVT migration and invasion (Figure 3A-3D), we hypothesized that RhoA degradation induced by a Par6/Smurf1/RhoA signaling might be attenuated upon T $\beta$ R3 knockdown. Thus, HTR cells were transfected with siCon or siT $\beta \mathrm{R} 3$, followed by TGF- $\beta 2$ stimulation and RhoA protein level assessment using Western blot analysis. A decrease in RhoA was readily detectable upon TGF- $\beta 2$ stimulation in siCon-transfected cells (Figure 3E, left panel, top blot, compare lane 2 to lane $1 ; 3 \mathrm{~F}$, left column, compare red bar to green bar). However, when T $\beta$ R3 protein level was reduced, a RhoA decrease was no longer observed (Figure 3E, right panel, top blot, compare lane 4 to lane $3 ; 3 \mathrm{~F}$, middle column). To further support a role for this pathway, RhoA decrease was not observed (Figure 3E, right panel, top blot, compare lane 6 to lane 5; 3F, right column) in cells where Par6 protein level was downregulated (Figure 3E, right panel, compare lane 2 to lane 1) using a siRNA specific against human Par6 [36]. Taken together, these results suggested that the Par6/ Smurf1/RhoA signaling is likely involved in the T $\beta$ R3mediated regulation of migration and invasion of EVT cells.

\section{The expression of $\mathrm{H} 19$ and T $\beta \mathrm{R} 3$ is decreased in FGR placentae}

Given that failure of trophoblast invasion is associated with FGR [7, 8] and that $\mathrm{H} 19$ positively regulates trophoblast cell migration and invasion through T $\beta R 3-$ mediated signaling (current work), we sought to determine whether the expression of H19 and T $\beta$ R 3 might be altered in human FGR placentae. Thus, placental tissue was collected from pregnancies with idiopathic preterm FGR $(n=12)$ and gestational age-matched controls with spontaneous preterm delivery $(n=12)$ (Patient characteristics in Supplementary Table S1). RNAs were isolated and gene expression levels were determined by RT-qPCR. Mann-Whitney $U$ test revealed a significant $\mathrm{H} 19$ decrease in the FGR placentae (FGR) compared to the control (CON) group (Figure 4A, $p=0.024$,). Not surprisingly, there was a parallel decrease in $\mathrm{T} \beta \mathrm{R} 3$ in the FGR compared to the CON group (Figure 4B,
A

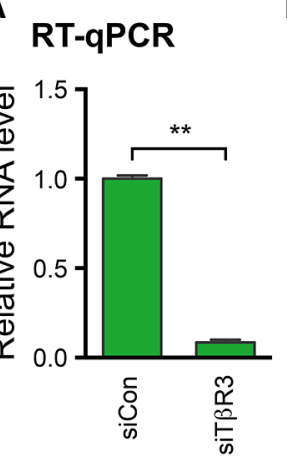

\section{Western}

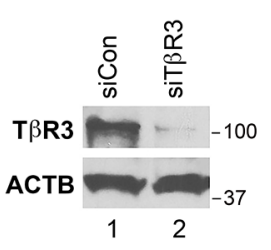

$E$

Western

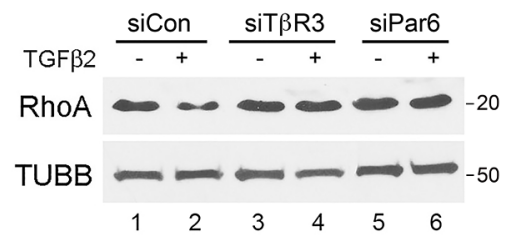

C

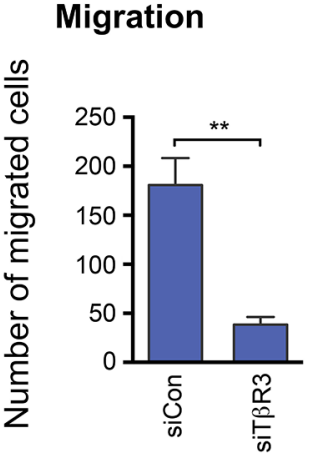

$\mathbf{F}$

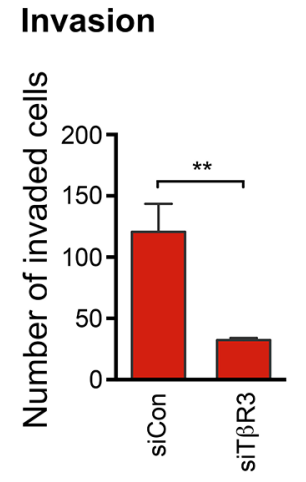

Western Quant

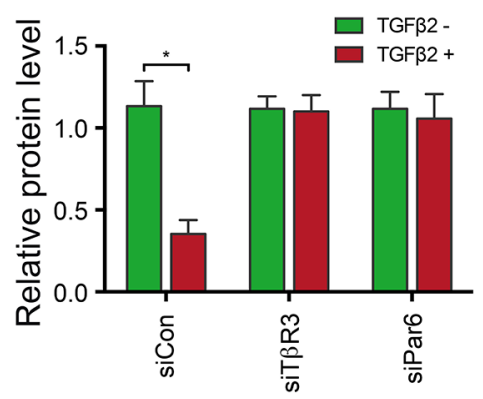

Figure 3: T $\beta R 3$ positively impacts EVT migration and invasion through a non-canonical signaling pathway. HTR cells were transfected with siCon or siT $\beta$ R3. T $\beta$ R3 RNA and protein levels were determined by RT-qPCR (A) and Western blot (B) analysis $48 \mathrm{~h}$ post-transfection. Protein molecular size markers in $\mathrm{kDa}$ are indicated on the right of the blots. Cell migration $(\mathbf{C})$ and invasion (D) were analyzed $48 \mathrm{~h}$ post-transfection. Numbers are mean $\pm \mathrm{SD}(n=3) .{ }^{* *} p<0.01$. (E) HTR cells were transfected with siCon, siT $\beta R 3$ or siPar6. In the left panel, at $48 \mathrm{~h}$ post-transfection, cells were treated with (+) or without (-)TGF- $\beta 2$ for $6 \mathrm{~h}$, followed by Western blot analysis to determine protein levels of RhoA (top blot) and beta-tubulin (bottom blot), which was used as a loading control. In the right panel, at $48 \mathrm{~h}$ post-transfection, proteins were extracted and Western blot analysis was carried out to determine Par6 protein levels. TUBB serves as a loading control. (F) Western blot quantifications using ImageJ from three independent transfection experiments are shown. One-sample $t$ tests were performed to compare each data point with the siCon control. Numbers are mean $\pm \operatorname{SD}(n=3) .{ }^{*} p<0.05$. 
$p=0.0145$, ). Spearman correlation showed a positive relationship between $\mathrm{H} 19$ and T $\beta \mathrm{R} 3$ (Figure 4C, $p<0.0001$ ), suggesting an in vivo functional interaction between H19 and T $\beta$ R3. Notably, there was no significant difference in the expression of T $\beta \mathrm{R} 1$ between the two groups (Figure 4D). Together, these results suggested that decreased expression of $\mathrm{H} 19$ and T $\beta \mathrm{R} 3$ might contribute to impaired migration and invasion of trophoblast cells during placental development in pregnancies complicated by FGR.

\section{A}

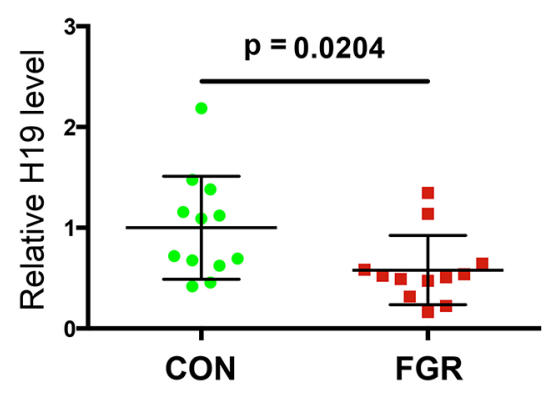

C
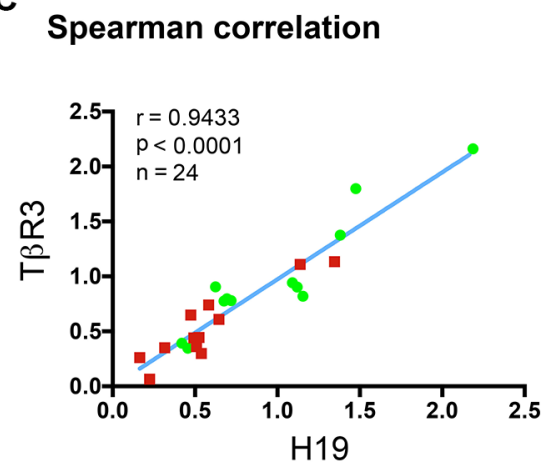

\section{DISCUSSION}

In the present study we show that $\mathrm{H} 19$ repression decreases TGF- $\beta$ signaling via the Par6/Smurf1/ RhoA pathway activated by T $\beta$ R3, leading to impaired migration and invasion of EVT cells (Figure 5). We provide evidence that dysregulation of H19/T $\beta R 3$ signaling may contribute to the underlying mechanism of idiopathic FGR.

B T $\beta$ R3

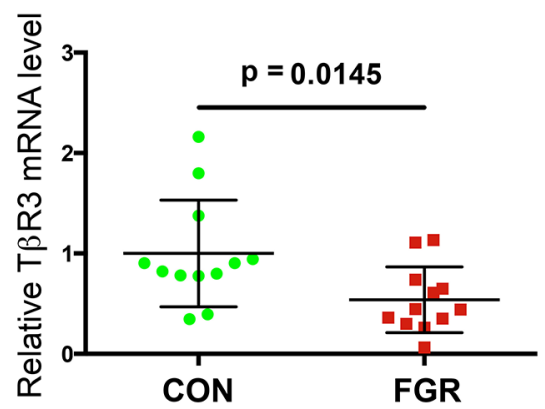

D T $\beta$ R1

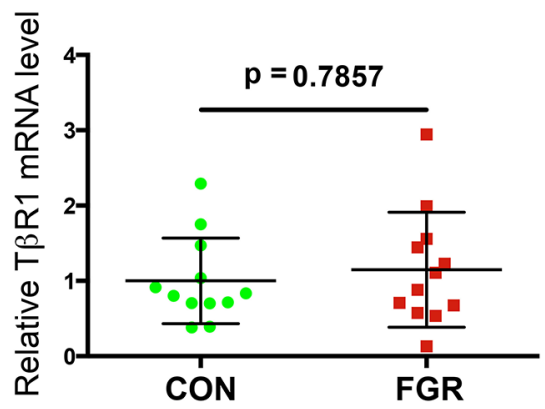

Figure 4: The expressions of H19 and T $\beta$ R3 are significantly decreased in the FGR placentae. (A, B, D) Scatter plot of RNA levels determined by RT-qPCR. The horizontal line represents group median, and the whiskers mark the interquartile range (C) Spearman correlation suggests an in vivo positive correlation between the expressions of H19 and T $\beta$ R3 in a statistically significant manner. Spearman correlation coefficient, $p$ values, and sample numbers are marked on the left top of the plot.

\section{H19}

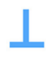

Let-7 $\uparrow$

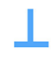

$\mathrm{T} \beta \mathrm{R} 3 \downarrow$

\section{Par6/Smurf1/RhoA $\downarrow$}

\section{EVT migration and invasion $\downarrow$}

Figure 5: A model of H19/TßR3-mediated signaling in the regulation of EVT migration and invasion. 
The role of different, individual TGF- $\beta$ cytokine isoforms in regulating EVT migration and invasion has been studied for many years (reviewed in [8, 27]). Recently, imbalanced TGF- $\beta$ signaling through two T $\beta$ R1 receptor subtypes (ALK5 and ALK1) has been suggested as a pathogenic cause of FGR [37]. In this work, the authors presented evidence for altered TGF- $\beta$ signaling via the canonical SMAD pathway, leading to an elevated sphingosine:ceramide ratio found in FGR placentae. They postulated that this finding explains the augmented trophoblast cell death rates and impaired placentation seen in FGR. However, they also noted that the canonical SMAD pathway does not entirely explain their findings and suggest a role for a non-redundant mechanism by which TGF- $\beta$ signaling is altered in cases of FGR. Our study uncovers a novel pathway, Par6/Smurf1/RhoA, which is activated by $T \beta R 3$ and regulates trophoblast cell migration and invasion. It is possible that both the canonical and the noncanonical pathways play critical and non-redundant roles in placentation. It remains to be investigated whether the two pathways cross-talk and whether they may act in concert to regulate trophoblast cell function.

While believing that T $\beta \mathrm{R} 3$ is one downstream mediator of the H19/let-7-mediated regulation, our RNA-seq data revealed numerous gene expression changes as a result of H19 knockdown (Supplementary Data 1). The partial rescue of T $\beta R 3$ expression by iLet7 (Figure 2A, middle column) versus the full restoration of migration and invasion to the siH19 cells by iLet7 (Figure 1A-1C) implicates other let-7-targeted genes in the H19-mediated regulation. The incomplete restoration of $\mathrm{T} \beta \mathrm{R} 3$ expression by iLet 7 was not surprising given that H19 is a multifunctional lncRNA. We have recently reported that $\mathrm{H} 19$ alters gene methylation genome-wide through modulation of the S-adenosylhomocysteine hydrolase activity [23], including H19 knockdown-induced hypomethylation of T $\beta$ R3 in the intron region [23]. It remains to be tested whether downregulation of H19 in human trophoblast cells might induce methylation changes in the T $\beta R 3$ gene, which may explain the partial restoration of T $\beta \mathrm{R} 3$ expression by iLet7. Our findings represent the first example of a lncRNA-based mechanism of FGR and hold promise for the development of novel predictive, diagnostic and therapeutic modalities for idiopathic FGR.

\section{MATERIALS AND METHODS}

\section{Materials}

HTR-8/SVneo cells were a generous gift from Charles Graham (Queen's University, Kingston, Canada) and were cultured in RPMI1640 (Gibco, 11965-092) supplemented with 10\% fetal bovine serum, heat inactivated, $1 \%$ penicillin/streptomycin, and $1 \%$ L-glutamine. Antibodies for T $\beta$ R3 (Abcam, ab166705; used at a dilution of 1/500), Par6 (Abcam, ab180159; used at a dilution of 1/1000), TUBB (Abcam, ab6046; used at a dilution of 1/10000), ACTB (Cell Signaling, 4967; used at a dilution of 1/10000), RhoA (Cell Signaling, 2117; used at a dilution of 1/1000), SMAD2/3 (Cell Signaling, 3102; used at a dilution of $1 / 1000)$, and phosphorylated SMAD2/3 (Cell Signaling, 3101; used at a dilution of 1/1000) were purchased. siH19 (Ambion, 4390816/n272452), siT $\beta$ R3 (Dharmacon, L-010545-00), siPar6 (Ambion, 4392420/ s27161), siCon (Ambion, Am4636), iLet7 (Ambion, 4392431), iCon (Ambion, AM17010), let-7 mimics (let7) (Ambion, AM17100/PM10050) and miCon (Ambion, AM17110) were purchased. Plasmids expressing human H19 (pH19) and empty vector were previously described $[14,16]$. TGF- $\beta 2$ cytokine was purchased (Cell Signaling, 8406).

\section{siRNA knockdown, iLet-7 rescue, and H19 overexpression experiments}

Cells were transfected in a 48 -well plate scale. To prepare siRNA transfection solution for each well, 15 pmol of siCon (or siH19, siPar6, siT $\beta$ R3) was mixed with $50 \mu \mathrm{l}$ OPTI-MEM by gentle pipetting. In parallel, $0.5 \mu \mathrm{l}$ Lipofectamine 2000 was mixed with $50 \mu$ OPTI-MEM. Following 5 minutes of incubation at room temperature (RT), the two were mixed by gentle pipetting and incubated for 20 to 30 minutes at RT to allow siRNA/lipid complexes to form. At the end of incubation, the $200 \mu \mathrm{l}$ transfection solution was used to re-suspend the cell pellet $\left(\sim 4 \times 10^{4}\right.$ cells/well). After incubation at RT for 10 minutes, regular growth medium was added at a ratio of 1:3 (1 volume of transfection solution/2 volumes of growth medium) and the cell suspension was transferred to the culture plate. After $24 \mathrm{~h}$ incubation at $37^{\circ} \mathrm{C}$ in $5 \% \mathrm{CO}_{2}$, the medium was replaced with fresh growth medium. RNAs and proteins were extracted and analyzed at the indicated time points following transfection.

Plasmid DNA transfections were carried out as described for siRNA, except that $0.8 \mu \mathrm{g}$ DNA in $50 \mu \mathrm{l}$ OPTI-MEM and $0.5 \mu$ l Lipofectamine 2000 in $50 \mu \mathrm{l}$ OPTIMEM were used for each well of cells (the $100 \mu \mathrm{l}$ of final transfection reagent with $400 \mu \mathrm{l}$ of regular growth medium were added to each well). For iLet7 rescue experiments, $15 \mathrm{pmol}$ of siCon/siH19 and $45 \mathrm{pmol}$ of iCon/iLet7 were used for each well of cells.

\section{RNA extraction and RT-qPCR}

Total RNAs were extracted from cells using PureLink RNA Mini Kit (Ambion, catalog number 12183018A). cDNA was synthesized using Bio-Rad iSCRIPT kit (1725122) in a $20 \mu \mathrm{l}$ reaction containing 100-500 ng of total RNA. Real-time quantitative PCR was performed in a $15 \mu \mathrm{l}$ reaction containing $0.5-1 \mu \mathrm{l}$ of cDNA using iQSYBRGreen (Bio-Rad) in a Bio-Rad iCycler. PCR was performed by initial denaturation at $95^{\circ} \mathrm{C}$ for $5 \mathrm{~min}$, followed by 40 cycles of $30 \mathrm{sec}$ at $95^{\circ} \mathrm{C}, 30 \mathrm{sec}$ at $60^{\circ} \mathrm{C}$, and $30 \mathrm{sec}$ at $72^{\circ} \mathrm{C}$. Specificity was verified by melting curve analysis and agarose gel electrophoresis. The 
threshold cycle $(\mathrm{Ct})$ values of each sample were used in the post-PCR data analysis. The real-time PCR primers are listed in Supplementary Table 2.

\section{Western blot analysis}

Cell pellets were quickly lysed in 5 volumes of $2 \times$ SDS-sample buffer heated at $100^{\circ} \mathrm{C}$ for $5 \mathrm{~min}$, with occasional vortexing. Five to $10 \mu \mathrm{l}$ of homogenized samples were loaded onto $10 \%$ SDS gel, followed by Western blot analysis. The linear dynamic range of each protein of interest was determined by serial dilutions. Bands on Western blot gels were quantified using ImageJ.

\section{Let-7 transfection and T $\beta R 3$ RNA and protein analysis}

To provide further evidence that $\mathrm{T} \beta \mathrm{R} 3$ is a target of let-7, let-7 mimics were transfected into HTR cells in a 48-well plate scale, followed by RNA and protein analyses $6 \mathrm{~h}$ and $48 \mathrm{~h}$ later, respectively. To analyze let-7 effects on RNA stability, let-7 transfection combined with actinomycin D time course analysis was performed. To prepare transfection cocktail, 1 pmol of control miRNA (miCon) or let-7a mimic was mixed with $50 \mu \mathrm{l}$ of OPTIMEM. In parallel, $0.5 \mu \mathrm{l}$ of Lipofectamine 2000 was mixed with $50 \mu \mathrm{l}$ of OPTI-MEM. Following 5 min of incubation, the two solutions were mixed and incubated at RT for 20 min. The resulting $100 \mu$ of transfection cocktail was added to HTR cells pre-washed with OPTI-MEM. Upon adding the transfection cocktail, actinomycin D was also added to each well at a final concentration of $10 \mu \mathrm{g} / \mathrm{ml}$. Total RNA was extracted at the indicated time points, followed by RTqPCR analysis. Results are presented after normalization against beta-tubulin mRNA levels with 0 time point RNA levels arbitrarily set as 1 . For T $\beta \mathrm{R} 3$ western blot analysis, cells harvested at $48 \mathrm{~h}$ post-transfection were lysed in 5 volumes of $2 \times \mathrm{SDS}$-sample buffer heated at $100^{\circ} \mathrm{C}$ for $5 \mathrm{~min}$. Five to $10 \mathrm{ml}$ of homogenized samples were loaded onto $10 \%$ SDS gel, followed by Western blot analysis as described above.

\section{Quantitative cell migration and invasion assays}

These were carried out as previously described [38] with minor modifications. Briefly, transwell chambers (Corning, 3422, 8- $\mu \mathrm{m}$ pores) placed into a 24-well plate (Fisher Scientific, 07200150,) were used in the assays. The lower chamber was filled with $500 \mu \mathrm{l}$ RPMI 1640 containing 20\% FBS. Cells were trypsinized, counted, and re suspended in serum-free RPMI. For migration assays, $5 \times 10^{4}$ cells in $200 \mu \mathrm{l}$ serum-free RPMI were added to the upper chamber. Equal numbers of cells from experimental and control groups were loaded into the upper chamber. The cells were allowed to migrate for $20 \mathrm{~h}$ at $37^{\circ} \mathrm{C}$ before fixing. For invasion assays, $100 \mu \mathrm{l}$ Matrigel (BD Biosciences, Bedford, MA, USA), 1:3 diluted in serum-free RPMI, was coated onto the upper chamber and incubated at $37^{\circ} \mathrm{C}$ for $2 \mathrm{~h}$. Equal numbers of cells from experimental and control groups were seeded into the upper chamber at a concentration of $8 \times 10^{4} / 200 \mu \mathrm{l}$ and incubated for $36 \mathrm{~h}$ at $37^{\circ} \mathrm{C}$ before fixing. The non-migrated cells were removed from the upper surface of the membrane by scraping with a cotton swab. Cells on the bottom surface of the membrane were fixed with $95 \%$ ethanol at RT for $30 \mathrm{~min}$, gently rinsed with phosphate-buffered saline (PBS), stained with Dapi for $5 \mathrm{~min}$, and photographed using a Zeiss (Melville, NY, USA) microscope system. Migration/invasion was assessed by counting the number of stained cell nuclei from 5 random fields per filter in each group at $\times 100$ magnification. The experiments were conducted in triplicate. Cell counts were expressed as the mean number of cells per field of view.

\section{Cell viability analysis}

To prepare for cell viability analysis, cells were seeded at $48 \mathrm{~h}$ post-transfection in 96-well plates at a density of $1 \times 10^{4} /$ well at the same time when migration and invasion assays were initiated. Cells were allowed to grow for $36 \mathrm{~h}$ and viabilities were determined using the Cell Titer Blue Cell Viability kit (Promega) according to the manufacturer's protocols.

\section{Cytokine treatment}

Cells were transfected with siCon, siT $\beta$ R3 or siPar6 in 48-well plates as described above. The medium was changed into serum-free RPMI 1640 at $24 \mathrm{~h}$ posttransfection. TGF- $\beta 2$ was applied to the cell $48 \mathrm{~h}$ posttransfection at a final concentration of $20 \mathrm{ng} / \mathrm{ml}$ in serumfree RPMI 1640. Six hours later, protein was extracted, followed by Western blot analysis.

\section{Study population}

We studied placenta samples from 24 women enrolled in the following groups:

idiopathic fetal growth restriction (FGR, $n=12$ ) and preterm control (CON, $n=12$ ). Placentae were collected under protocols approved by the Human Investigation Committee of Yale University. Informed consent was obtained from all participants prior to enrollment. Gestational age was established based on menstrual dating confirmed by sonographic examination prior to 20 weeks' of gestation. Characteristics of the study population are shown in Supplementary Table 2.

The CON group consisted of placentae from pregnancies with spontaneous preterm labor and/or preterm premature rupture of membranes without evidence of clinical nor histological chorioamnionitis. FGR was defined as weight $<10$ th percentile for gestational age at birth in pregnancies not complicated by preeclampsia. A detailed description of these patients was previously reported [39-41]. 


\section{Tissue collection and analysis of gene expression}

Following delivery, placentae were brought immediately to the laboratory. Approximately $1 \mathrm{~g}$ of placental villous tissue from the central region of the placenta (dissected free from decidua basalis) was collected, frozen in liquid nitrogen, and maintained at $-80^{\circ} \mathrm{C}$. RNA was extracted from placental tissue using Trizol reagent (Life Technologies, Grand Island, NY). cDNA was synthesized from 3-5 $\mu \mathrm{g}$ RNA using oligo-deoxythymidine 12-18 (catalog item 18418-012; Invitrogen) and SuperScript II reverse transcriptase (catalog item 18064-014; Invitrogen) in a $20 \mu \mathrm{l}$ reaction volume according to the manufacturer's instructions. qPCR was performed as described above using beta-tubulin mRNA as a loading control.

\section{Statistical analysis}

Patient characteristics and in vivo gene expression data are presented as median and interquartile range (IQR) and were analyzed using Mann-Whitney $U$-test. Spearman correlations were performed for gene co-expression analyses. In vitro data are presented as mean \pm standard deviation (SD) and analyzed using two-tailed Student $t$ test. Statistical analyses were performed using the Statistical Package for the Social Science (SPSS) computer software version 17.0 (IBM SPSS Statistics, Chicago, IL, USA). Figures were constructed using Prism 6 version 6.0f (GraphPad Software, Inc.). $P$-values $<0.05$ were considered significant.

\section{ACKNOWLEDGMENTS}

We thank Charles Graham for the generous gift of HTR-8/SVneo cells. This work was supported by grants from Bennack-Polan Foundation and Natural Science Foundation of China 81428018 to Y.H.

\section{CONFLICTS OF INTEREST}

All authors declare no conflicts of interest related to this work.

\section{Authors' contributions}

L.Z. and Y.H. designed the study. L.Z., J.L.,Y.M. and T.G. performed experiments and analyzed data. C.S.B., I.A.B. and S.G. provided human placental cDNA. L.L. did bioinformatics analysis of the RNA-seq data. R.B., C.S.B., I.A.B., S.G. and M.P. provided intellectual insights into FGR and critical reading of the manuscript. Y.H. and L.Z. wrote the manuscript.

\section{REFERENCES}

1. Harkness UF, Mari G. Diagnosis and management of intrauterine growth restriction. Clin Perinatol. 2004; 31:743-64, vi.
2. American College of, O. and Gynecologists, ACOG Practice bulletin no. 134: fetal growth restriction. Obstet Gynecol. 2013; 121:1122-33.

3. Gilbert WM, Danielsen B. Pregnancy outcomes associated with intrauterine growth restriction. Am J Obstet Gynecol. 2003; 188:1596-9; discussion 1599-601.

4. Kanaka-Gantenbein C, Mastorakos G, Chrousos GP. Endocrine-related causes and consequences of intrauterine growth retardation. Ann N Y Acad Sci. 2003; 997:50-7.

5. Larroque B, Bertrais S, Czernichow P, Leger J. School difficulties in 20-year-olds who were born small for gestational age at term in a regional cohort study. Pediatrics. 2001; 108:111-5.

6. Strauss RS. Adult functional outcome of those born small for gestational age: twenty-six-year follow-up of the 1970 British Birth Cohort. JAMA. 2000; 283:625-32.

7. Doridot L, Miralles F, Barbaux S, Vaiman D. Trophoblasts, invasion, and microRNA. Front Genet. 2013; 4:248.

8. Lash GE. Molecular Cross-Talk at the Feto-Maternal Interface. Cold Spring Harb Perspect Med, 2015; 10.1101/ cshperspect.a023010.

9. Gabory A, Jammes H, Dandolo L. The H19 locus: role of an imprinted non-coding RNA in growth and development. Bioessays. 2010; 32:473-80.

10. Monnier P, Martinet C, Pontis J, Stancheva I, Ait-Si-Ali S, Dandolo L. H19 lncRNA controls gene expression of the Imprinted Gene Network by recruiting MBD1. Proc Natl Acad Sci U S A. 2014; 110:20693-20698.

11. Keniry A, Oxley D, Monnier P, Kyba M, Dandolo L, Smits G, Reik W. The H19 lincRNA is a developmental reservoir of miR-675 that suppresses growth and Igflr. Nat Cell Biol. 2012; 14:659-65.

12. Venkatraman A, He XC, Thorvaldsen JL, Sugimura R, Perry JM, Tao F, Zhao M, Christenson MK, Sanchez R, Yu JY, Peng L, Haug JS, Paulson A, et al. Maternal imprinting at the H19-Igf2 locus maintains adult haematopoietic stem cell quiescence. Nature. 2013; 500:345-9.

13. Dey BK, Pfeifer K, Dutta A. The H19 long noncoding RNA gives rise to microRNAs miR-675-3p and miR-675-5p to promote skeletal muscle differentiation and regeneration. Genes \& Dev. 2014; 28:491-501.

14. Kallen AN, Zhou XB, Xu J, Qiao C, Ma J, Yan L, Lu L, Liu C, Yi JS, Zhang H, Min W, Bennett AM, Gregory RI, et al. The imprinted H19 lncRNA antagonizes let-7 microRNAs. Mol Cell. 2013; 52:101-12.

15. Gao Y, Wu F, Zhou J, Yan L, Jurczak MJ, Lee HY, Yang L, Mueller M, Zhou XB, Dandolo L, Szendroedi J, Roden M, Flannery C, et al. The H19/let-7 double-negative feedback loop contributes to glucose metabolism in muscle cells. Nucleic Acids Res. 2014; 42:13799-811.

16. Yan L, Zhou J, Gao Y, Ghazal S, Lu L, Bellone S, Yang Y, Liu N, Zhao X, Santin AD, Taylor H, Huang Y. Regulation of tumor cell migration and invasion by the H19/let-7 axis 
is antagonized by metformin-induced DNA methylation. Oncogene. 2015; 34:3076-84.

17. Ghazal S, McKinnon B, Zhou J, Mueller M, Men Y, Yang L, Mueller M, Flannery C, Huang Y, Taylor HS. H19 lncRNA alters stromal cell growth via IGF signaling in the endometrium of women with endometriosis. EMBO Mol Med. 2015; 7:996-1003.

18. Gao WL, Liu M, Yang Y, Yang H, Liao Q, Bai Y, Li YX, Li D, Peng C, Wang YL. The imprinted H19 gene regulates human placental trophoblast cell proliferation via encoding miR-675 that targets Nodal Modulator 1 (NOMO1). RNA Biol. 2012; 9.

19. Kappil MA, Green BB, Armstrong DA, Sharp AJ, Lambertini L, Marsit CJ, Chen J. Placental Expression Profile of Imprinted Genes Impacts Birth Weight. Epigenetics. 2015; 10:842-9.

20. Buckberry S, Bianco-Miotto T, Roberts CT. Imprinted and X-linked non-coding RNAs as potential regulators of human placental function. Epigenetics. 2014; 9:81-9.

21. Ariel I, Lustig O, Oyer CE, Elkin M, Gonik B, Rachmilewitz J, Biran H, Goshen R, de Groot N, Hochberg A. Relaxation of imprinting in trophoblastic disease. Gynecol Oncol. 1994; 53:212-9.

22. Graham $\mathrm{CH}$, Hawley TS, Hawley RG, MacDougall JR, Kerbel RS, Khoo N, Lala PK. Establishment and characterization of first trimester human trophoblast cells with extended lifespan. Exp Cell Res. 1993; 206:204-11.

23. Zhou J, Yang L, Zhong T, Mueller M, Men Y, Zhang N, Xie J, Giang K, Chung H, Sun X, Lu L, Carmichael GG, Taylor HS, et al. H19 lncRNA alters DNA methylation genome-wide by regulating S-adenosylhomocysteine hydrolase. Nat Commun. 2015; 6:10221.

24. Huang T, David L, Mendoza V, Yang Y, Villarreal M, De K, Sun L, Fang X, Lopez-Casillas F, Wrana JL, Hinck AP. TGF- $\beta$ signalling is mediated by two autonomously functioning TbetaRI:TbetaRII pairs. EMBO J. 2011; 30:1263-76.

25. Kretzschmar M, Massague J. SMADs: mediators and regulators of TGF- $\beta$ signaling. Curr Opin Genet Dev, 1998; 8:103-11.

26. Lopez-Casillas, F, Wrana JL, Massague J. Betaglycan presents ligand to the TGF $\beta$ signaling receptor. Cell. 1993; 73:1435-44.

27. Knofler M. Critical growth factors and signalling pathways controlling human trophoblast invasion. Int J Dev Biol. 2010; 54:269-80.

28. Mitchell EJ, Fitz-Gibbon L, O'Connor-McCourt MD. Subtypes of betaglycan and of type I and type II transforming growth factor-beta (TGF- $\beta$ ) receptors with different affinities for TGF- $\beta 1$ and TGF- $\beta 2$ are exhibited by human placental trophoblast cells. J Cell Physiol. 1992; 150:334-43.

29. Sanchez NS, Barnett JV. TGF $\beta$ and BMP-2 regulate epicardial cell invasion via TGF $\beta$ R3 activation of the Par6/ Smurf1/RhoA pathway. Cell Signal. 2012; 24:539-48.
30. Sanchez NS, Hill CR, Love JD, Soslow JH, Craig E, Austin AF, Brown CB, Czirok A, Camenisch TD, Barnett JV. The cytoplasmic domain of TGF $\beta$ R 3 through its interaction with the scaffolding protein, GIPC, directs epicardial cell behavior. Dev Biol. 2011; 358:331-43.

31. Fabian MR, Sonenberg N. The mechanics of miRNAmediated gene silencing: a look under the hood of miRISC. Nat Struct Mol Biol. 2012; 19:586-93.

32. Pal A, Huang W, Toy KA, Kleer CG. CCN6 knockdown disrupts acinar organization of breast cells in threedimensional cultures through up-regulation of type III TGF- $\beta$ receptor. Neoplasia. 2012; 14:1067-74.

33. Wang HR, Zhang Y, Ozdamar B, Ogunjimi AA, Alexandrova E, Thomsen GH, Wrana JL. Regulation of cell polarity and protrusion formation by targeting RhoA for degradation. Science. 2003; 302:1775-9.

34. Ozdamar B, Bose R, Barrios-Rodiles M, Wang HR, Zhang Y, Wrana JL. Regulation of the polarity protein Par6 by TGF $\beta$ receptors controls epithelial cell plasticity. Science. 2005; 307:1603-9.

35. Ridley AJ. RhoA, RhoB and RhoC have different roles in cancer cell migration. J Microsc. 2013; 251:242-9.

36. Sivasubramaniyam T, Garcia J, Tagliaferro A, MellandSmith M, Chauvin S, Post M, Todros T, Caniggia I. Where polarity meets fusion: role of Par6 in trophoblast differentiation during placental development and preeclampsia. Endocrinology. 2013; 154:1296-309.

37. Chauvin S, Yinon Y, Xu J, Ermini L, Sallais J, Tagliaferro A, Todros T, Post M, Caniggia I. Aberrant TGF $\beta$ Signalling Contributes to Dysregulation of Sphingolipid Metabolism in Intrauterine Growth Restriction. J Clin Endocrinol Metab. 2015; 100:E986-96.

38. Yin G, Alvero AB, Craveiro V, Holmberg JC, Fu HH, Montagna MK, Yang Y, Chefetz-Menaker I, Nuti S, Rossi M, Silasi DA, Rutherford T, Mor G. Constitutive proteasomal degradation of TWIST-1 in epithelial-ovarian cancer stem cells impacts differentiation and metastatic potential. Oncogene. 2013; 32:39-49.

39. Tang Z, Buhimschi IA, Buhimschi CS, Tadesse S, Norwitz E, Niven-Fairchild T, Huang ST, Guller S. Decreased levels of folate receptor-beta and reduced numbers of fetal macrophages (Hofbauer cells) in placentas from pregnancies with severe pre-eclampsia. Am J Reprod Immunol. 2013; 70:104-15.

40. Tang Z, Niven-Fairchild T, Tadesse S, Norwitz ER, Buhimschi CS, Buhimschi IA, Guller S. Glucocorticoids enhance CD163 expression in placental Hofbauer cells. Endocrinology. 2013; 154:471-82.

41. Guller S, Buhimschi CS, Ma YY, Huang ST, Yang L, Kuczynski E, Zambrano E, Lockwood CJ, Buhimschi IA. Placental expression of ceruloplasmin in pregnancies complicated by severe preeclampsia. Lab Invest. 2008; 88:1057-67. 